\title{
Терагерцовая фотопроводимость в графене в магнитном поле
}

\author{
() Ю.Б. Васильев ${ }^{1}$, С.Н. Новиков ${ }^{2}$, С.Н. Данилов ${ }^{3}$, С.Д. Ганичев ${ }^{3,4}$ \\ ${ }^{1}$ Физико-технический институт им. А.Ф. Иоффре Российской академии наук, \\ 194021 Санкт-Петербург, Россия \\ ${ }^{2}$ Aalto University, Tietotie 3, \\ 02150 Espoo, Finland \\ ${ }^{3}$ Terahertz Center TerZ, University of Regensburg, \\ Regensburg, Germany \\ ${ }^{4}$ CENTERA Laboratories, Institute of High Pressure Physics, Polish Academy of Sciences, \\ 01-142 Warsaw, Poland \\ E-mail: yu.vasiliev@mail.ioffe.ru
}

Поступила в Редакцию 28 ноября 2019 г.

В окончательной редакции 5 декабря 2019 г.

Принята к публикации 5 декабря 2019 г.

\begin{abstract}
Изучается терагерцовая фотопроводимость в магнитном поле в эпитаксиальном графене, выращенном на подложке $\mathrm{SiC}$. Проведены исследования зависимости амплитуды сигнала фотоотклика от величины магнитного поля при разных значениях концентрации электронов, тока смещения, интенсивности терагерцового излучения. Механизм фотопроводимости, основанный на нагреве электронов терагерцовым излучением, хорошо объясняет экспериментальные результаты. С увеличением величины магнитного поля обнаружен сильный рост сигнала фотопроводимости из-за увеличения времени релаксации вследствие подавления электрон-электронного рассеяния.
\end{abstract}

Ключевые слова: графен, фотопроводимость, терагерцовое излучение, магнитное поле.

DOI: 10.21883/FTP.2020.04.49146.9322

\section{1. Введение}

Благодаря своим уникальным свойствам, графен является перспективным материалом для создания быстродействующих приемников в широкой области спектра [1]. Особенно интересным является его применение для создания приборов в малоосвоенном терагерцовом (ТГц) диапазоне. Ожидалось, что графен мог бы использоваться для создания ТГц болометров на горячих электронах, поскольку электроны быстро нагреваются при поглощении света из-за небольшой электронной теплоемкости [2]. Однако оказалось, что подвижность носителей в графене слабо зависит от температуры, поэтому разогрев электронов при однородной засветке поверхности образца приводит к незначительному изменению его сопротивления. Экспериментально наблюдаемый болометрический сигнал в графене, как правило, слишком слабый, чтобы использовать чистый графен в качестве ТГц болометра. Увеличить температурный коэффициент сопротивления можно, например, за счет локализации носителей заряда. Так, в графене с сильным беспорядком [3] удалось зарегистрировать достаточно сильный болометрический сигнал. Ранее нами была измерена фотопроводимость в образцах графена, изготовленных в форме меандра [4]. Было показано, что сильная температурная зависимость сопротивления в таких образцах является следствием эффекта слабой локализации [5]. До настоящего времени подробного исследования ТГц фотопроводимости в таких приемниках не проводилось.

В данной работе исследуются зависимости величины ТГц фотопроводимости в эпитаксиальном графене от величины магнитного поля при разных значениях концентрации электронов, тока смещения, интенсивности терагерцового излучения. Нами обнаружен сильный, более чем на порядок, рост сигнала фотопроводимости с увеличением магнитного поля. Увеличение фотопроводимости в магнитном поле объясняется увеличением времени электрон-электронного рассеяния из-за неэквидистантности уровней Ландау в графене.

\section{2. Методика эксперимента}

Эксперименты проводились на структурах графена, полученных сублимацией на поверхности $\mathrm{Si}$ подложки 4H-SiC термическим разложением в атмосфере аргона. $\mathrm{C}$ помощью лазерной литографии образцам придавалась форма меандра, как показано на рис. 1, $a$. Измерения проводились на двух образцах шириной $W=25$ мкм и длиной $L=15$ мм, так что отношение $L / W=600$. Каждый образец имеет несколько контактов Тi/Au 5/50 нм, которые были сделаны электронным испарением. Измерения проводились для разных пар контактов, которые расположены как близко друг к другу, так и на большом расстоянии друг от друга.

В качестве источника терагерцового излучения использовался импульсный молекулярный лазер [6-8] с оптической накачкой TEA- $\mathrm{CO}_{2}$-лазером. Использование в качестве активной среды $\mathrm{NH}_{3}$ позволило работать на длинах волн 280, 148 и 90 мкм, что соответствует частотам 1.1, 2.0 и 3.3 ТГц. Импульсы длительностью $\sim 100$ нс имели пиковую мощность порядка десятков кВт, которая контролировалась с помощью детекторов на эффекте 


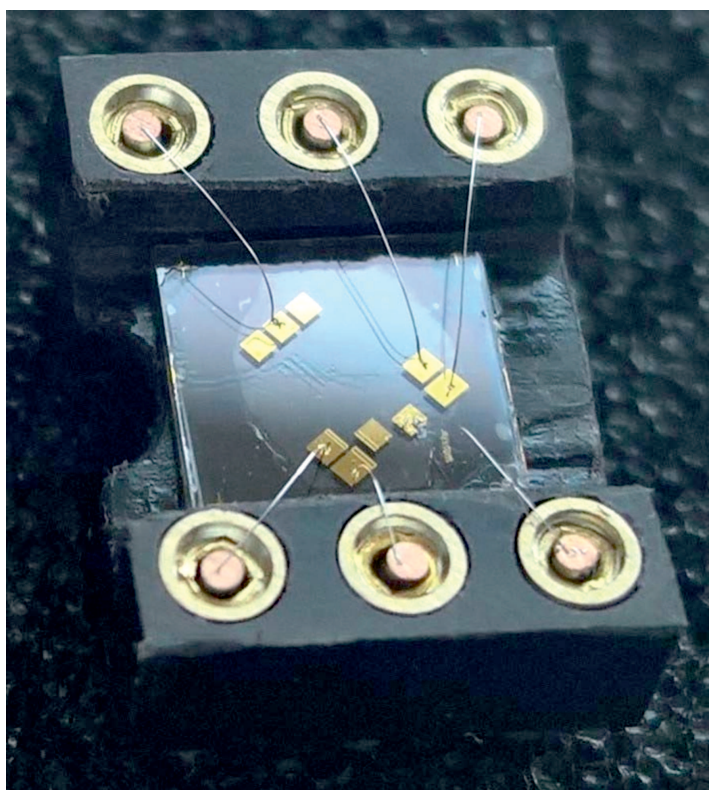

$b$

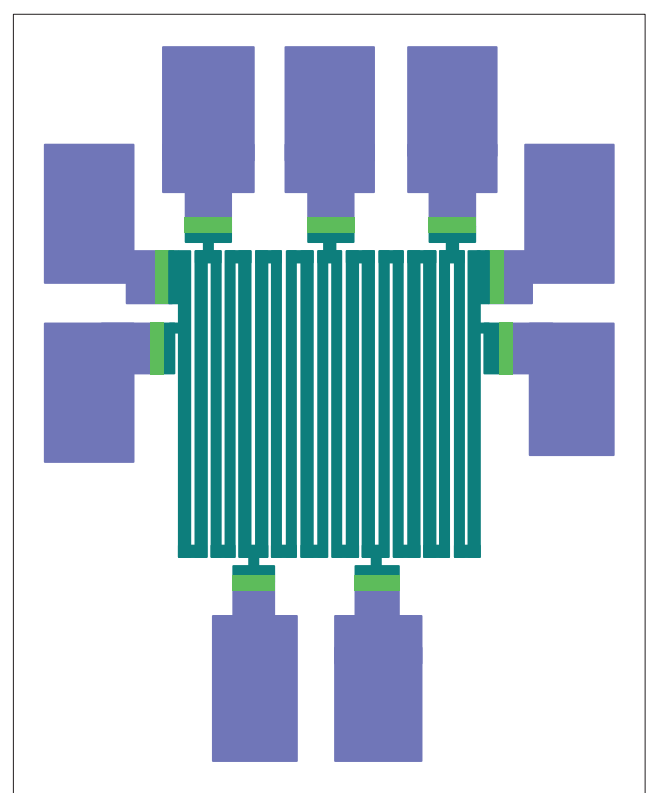

Рис. 1. $a-$ фотография образца, $b-$ форма образца в виде меандра, в котором ширина полоски равна 25 мкм и общая длина равна 15 мм.
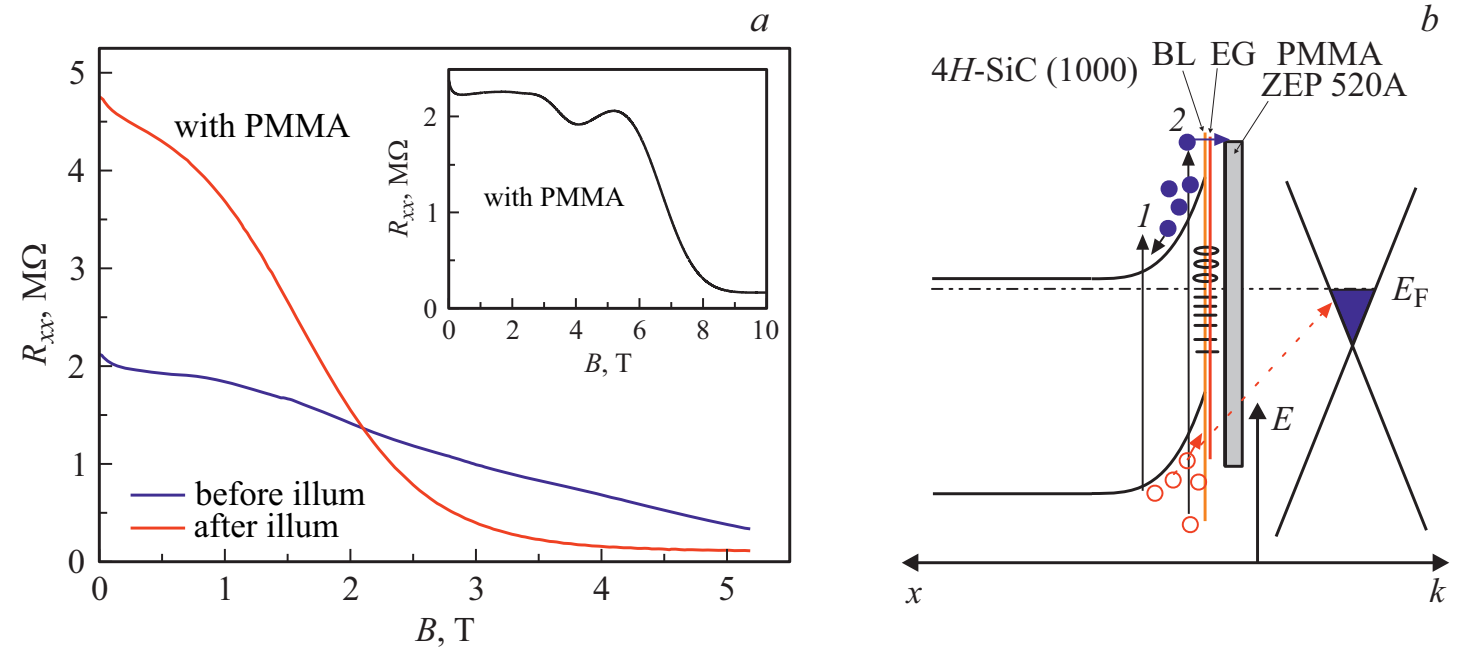

Рис. 2. $a$ - зависимости магнетосопротивления до и после засветки УФ, соответствующие разным концентрациям электронов в графене. На вставке - зависимость магнетосопротивления до нанесения полимерной пленки на поверхность графена, видны минимумы осцилляции Шубникова-де Гааза при больших факторах заполнения 6 и 10. $b$ - схематическое изображение зонной структуры образца, объясняющее механизм изменения концентрации электронов при засветке образца УФ светом. Обозначения на графике: BL - буферный слой, EG - слой эпитаксиального графена, PMMA ZEP520A — полимерная пленка.

увлечения электронов фотонами [9]. Профиль терагерцового излучения, измеренный пироэлектрической камерой, имеет почти гауссов профиль $\sim 1.5-3$ мм в диаметре, что соответствует интенсивности излучения $5-200 \kappa$ Кт $/ \mathrm{cm}^{2}$ в зависимости от длины волны излучения [10]. Измерялся сигнал фотопроводимости при нормальном падении терагерцового излучения. Внешнее магнитное поле величиной до 5.5 Тл прикладывалось нормально к поверхности образца и параллельно распространению излучения. Все измерения проводились при температуре жидкого гелия $T=4.2 \mathrm{~K}$.

\section{3. Изменение концентрации электронов в графене при облучении УФ излучением}

После изготовления структур в форме меандра поверхность образцов покрывалась последовательно полимерными пленками PMMA и ZEP520A для изменения концентрации носителей при облучении образцов ультрафиолетовым излучением. На рис. 2 представлены зависимости сопротивления образца от магнитного 

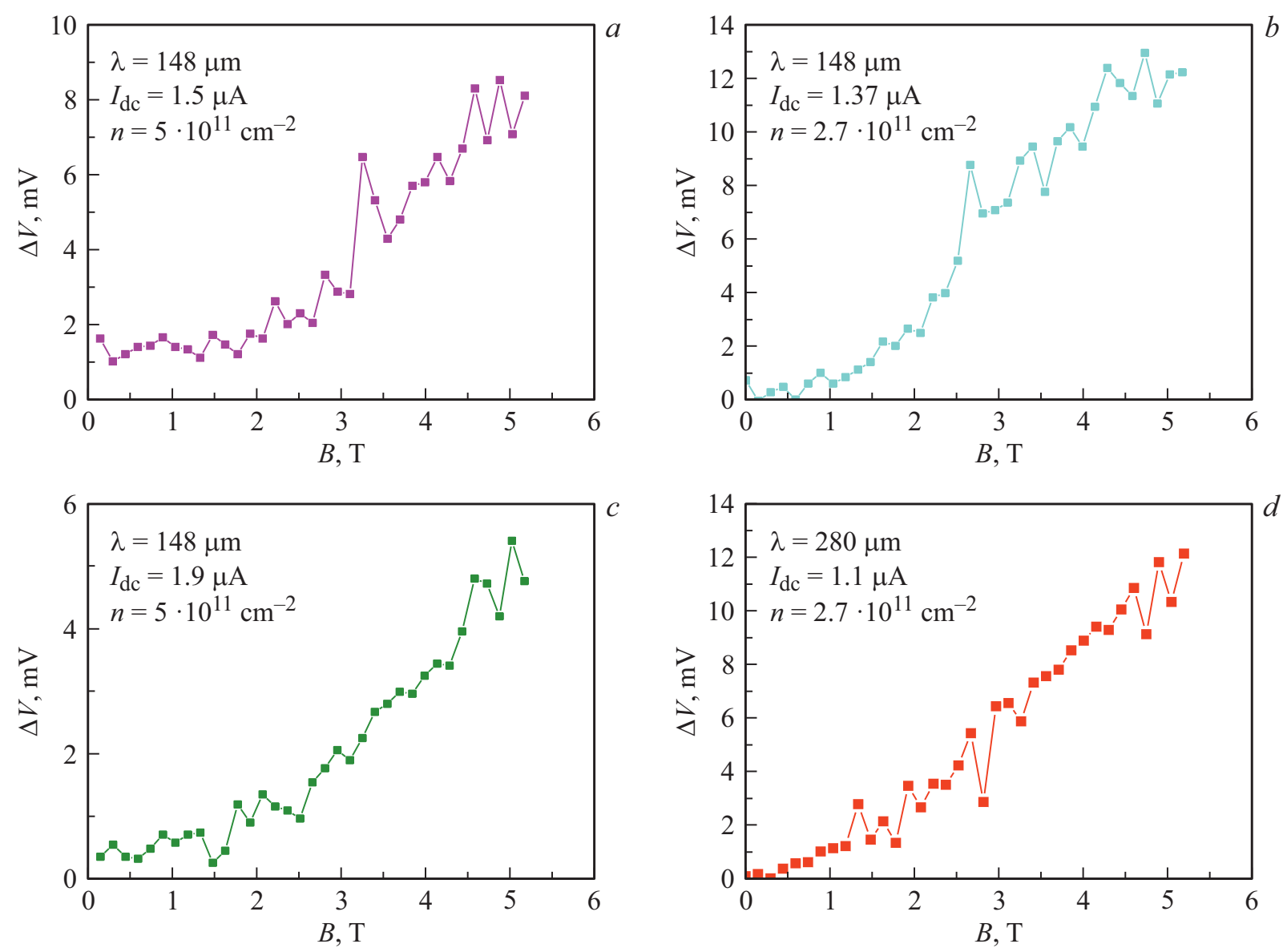

Рис. 3. Зависимости амплитуды сигнала фотопроводимости $\Delta V$ от магнитного поля для разных концентраций электронов (a) и $(b)$, токов смещения $(a)$ и $(c)$ и различных длин волн падающего излучения $(b)$ и $(d)$.

поля, измеренные до (синяя) и после (красная) засветки ультрафиолетовым светом, а также до нанесения пленки (см. вставку на рис. 2). До засветки концентрация электронов в графене составляла $5 \cdot 10^{11} \mathrm{~cm}^{-2}$. После засветки концентрация понизилась до величины $2.7 \cdot 10^{11} \mathrm{~cm}^{-2}$. Эффект изменения концентрации электронов под действием ультрафиолетового света был ранее изучен в ряде работ $[11,12]$. Отметим, что пленка из полимера ZEP520A, нанесенная на поверхность образца, отрицательно заряжается под действием УФ и служит в качестве отрицательно заряженного затвора. Меняя дозу облучения (длительность и интенсивность), можно управлять концентрацией электронов в графене в широких пределах. Схематическое изображение структуры, поясняющее механизм изменения концентрации под действием УФ, представлено на рис. 2, $b$.

\section{4. Результаты измерений фотопроводимости}

Результаты измерений фотопроводимости для монослойного эпитаксиального графена показаны на рис. 3 и 4. На рис. 3 показаны зависимости величины фотопроводимости $\Delta V$ от магнитного поля $B$ для разных длин волн излучения $\lambda$, токов смещения $I_{d c}$ и концентраций электронов $n$.

На рис. 3, $а$ показаны зависимости величины фотоотклика от магнитного поля при использовании излучения длиной волны, равной 148 мкм (частота 2 ТГц), и для концентрации носителей $n=5 \cdot 10^{11} \mathrm{~cm}^{-2}$. Четко видны два участка на этой зависимости. Вначале с ростом магнитного поля от 0 до 2 Тл интенсивность сигнала практически остается постоянной. При дальнейшем увеличении магнитного поля, при его значениях > 2 Тл наблюдается линейный рост сигнала с магнитным полем. При увеличении магнитного поля от 2 до 5 Тл амплитуда сигнала возрастает более чем в 4 раза. Для всех измеренных значений длин волн, токов смещения и концентраций наблюдаются аналогичные зависимости, а именно линейный рост амплитуды фотоответа с ростом магнитного поля возникает после участка в слабых полях, где сигнал слабо зависит от магнитного поля. Величина магнитного поля, после которого начинается рост сигнала, уменьшается с уменьшением концентрации носителей. Это следует из сравнения результатов, представленных на рис. 3, $a$ и $b$. Для концентрации носителей $n=2.7 \cdot 10^{11} \mathrm{~cm}^{-2}$ рост сигнала начинается с полей $\sim 1$ Тл. Как видно на рис. 3 , снижение концентрации приводит к уменьшению величины магнитного поля, при 

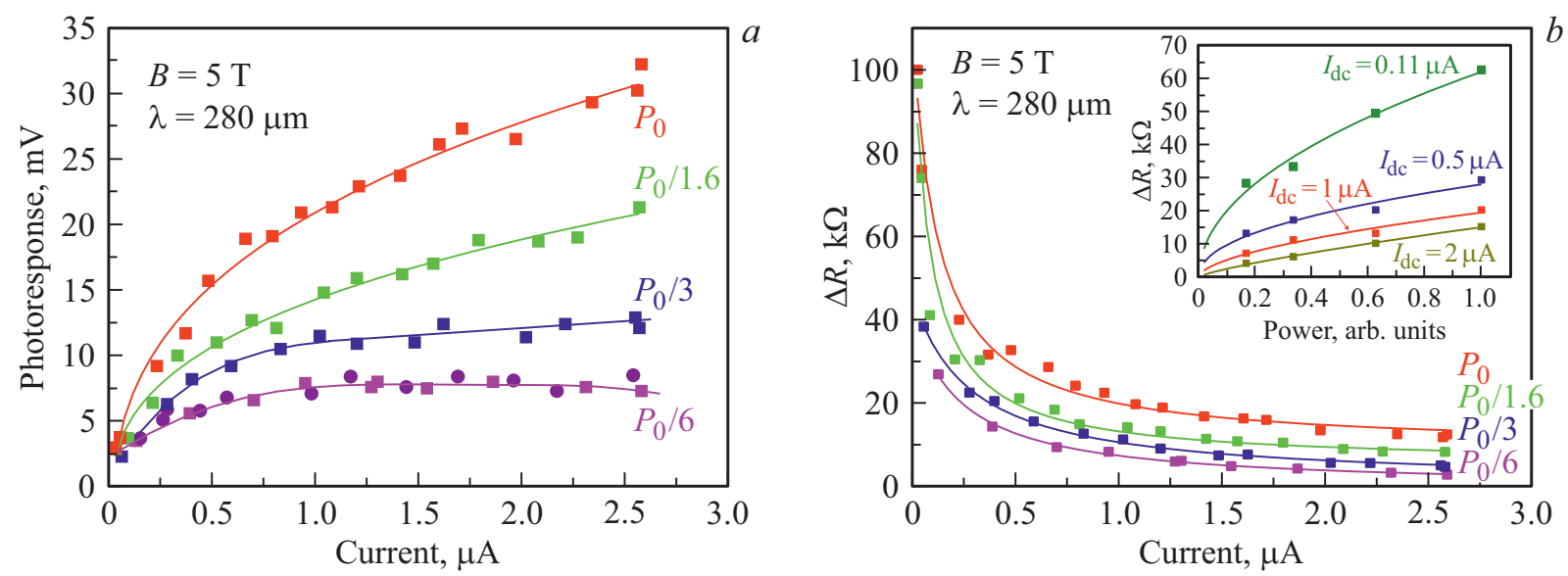

Рис. 4. $a-$ зависимости амплитуды сигнала фотопроводимости $\Delta V$. Показаны зависимости для излучения длиной 280 мкм при разных значениях мощности терагерцового излучения в магнитном поле $B=5$ Тл. Круги и квадраты для мощности $P_{0} / 6$ соответствуют измерениям, выполненным на разных контактах. $b$ - зависимости соответствующего изменения сопротивления образца $\Delta R$ под действием импульсов терагерцового излучения, как функции тока смещения. На вставке показана зависимость $\Delta R$ от мощности падающего излучения для $I_{d c}=0.11,0.5,1$ и 2 мкА.

котором начинается линейный рост фотопроводимости. На рис. 3, результаты аналогичных измерений представлены для большей величины тока смещения. Величина тока смещения не влияет на величину магнитного поля, после которого начинается рост фотопроводимости, но влияет на амплитуду фотопроводимости. С увеличением тока от 1.5 до 1.9 мкА амплитуда фотопроводимости в слабых полях уменышается от 1.7 до $\sim 0.5 \mathrm{MB}$.

На рис. 4 показаны зависимости амплитуды фотоответа $\Delta V$ и соответствующее изменение сопротивления образца $\Delta R=\Delta V / I_{d c}$ от величины постоянного тока смещения $I_{d c}$ для разных интенсивностей света. Сигнал фотопроводимости на рис. $4, a$ увеличивается с током $I_{d c}$ и достигает значений 30 мВ при максимальных использовавшихся токах $I_{d c}=2.7$ мкА. На рис. 4, $a$ представлены зависимости сигнала фотопроводимости от тока смещения при разных значениях мощности терагерцового излучения с длиной волны 280 мкм в магнитном поле $B=5$ Тл. Интенсивность излучения лазера изменялась в 6 раз. Видно, что сигнал зависит от тока нелинейно, и при наименьшей из использовавшихся мощностей лазера фотопроводимость насыщается при токах, превышающих 1 мкА. На рис. 4, $b$ отложены зависимости изменения сопротивления образца от тока смещения $\Delta R=\Delta V / I_{d c}$. Изменение сопротивления соответствует уменьшению сопротивлению графена под действием излучения. Для всех использовавшихся мощностей наибольшее изменение сопротивления графена происходит при наименьших токах. С ростом тока изменение сопротивления уменьшается в $\sim 5$ раз и становится независимым от тока.

\section{5. Обсуждение результатов}

Обратимся к обсуждению механизма фотоотклика в графене под действием терагерцового излучения при однородной засветке поверхности образца. В общем случае фотопроводимость может быть связана либо с изменением числа носителей в образце под действием света, либо с болометрическим эффектом, если разогрев электронного газа при поглощении излучения приводит к изменению подвижности носителей. Поскольку энергия Ферми в наших образцах значительно превышает энергию квантов терагерцового излучения, вертикальные оптические переходы между валентной зоной и зоной проводимости запрещены. Поэтому число носителей не меняется. Также можно пренебречь разогревом решетки графена в силу слабого поглощения излучения в решетке из-за атомарно-тонкого слоя графена. Наблюдаемый сигнал фотопроводимости объясняется разогревом электронов терагерцовым излучением. В отсутствие магнитного поля этот механизм был изучен в работе [4].

Экспериментальные результаты можно описать при помощи простой модели болометрического механизма фотопроводимости [2,13]. После поглощения квантов света электронная система нагревается до некоторой эффективной температуры $T_{e}=T_{L}+\Delta T_{e}$, которая превышает равновесную температуру (температуру решетки) $T_{L}$ на величину $\Delta T_{e}=P \tau / C_{e}$. Здесь $P-$ скорость поглощения энергии на единицу площади, $C_{e}-$ удельная теплоемкость электронного газа на единицу площади, $\tau$ - время релаксации (рассеяния) фотовозбужденных носителей. Величину фотопроводимости $\Delta V$ можно представить в виде

$$
\Delta V=I_{d c} \Delta R_{x x}=I_{d c} \frac{L}{W} \Delta \rho_{x x}=I_{d c} \frac{L}{W} \frac{\partial \rho_{x x}}{\partial T_{L}} \Delta T_{e},
$$

где $\Delta R_{x x}=\frac{\partial R_{x x}}{\partial T_{L}}=\Delta T_{e}$ - изменение сопротивления образца под действием излучения, $\frac{\partial \rho_{x x}}{\partial T_{L}}-$ температурный коэффициент удельного сопротивления, $L$ и $W$ - длина и ширина образца соответственно. Величина сигнала 
фотоотклика зависит от тока смещения $I_{d c}$ и пропорциональна отношению длины образца к его ширине $L / W$. Поэтому увеличение соотношения $L / W$ приводит к росту величины сигнала фотопроводимости при условии, что фотоответ вызван изменением удельного сопротивления $\Delta \rho_{x x}$. Этим обстоятельством объясняется выбор формы образцов в наших экспериментах.

Поскольку разогрев электронного газа $\Delta T_{e}=P \tau / C_{e}$ и, соответственно, сигнал фотопроводимости пропорциональны времени рассеяния, то величина фотопроводимости зависит от механизмов рассеяния электронов. Процессы релаксации горячих носителей в графене изучались в многочисленных работах как в нулевом магнитном поле $[14,15]$, так и при наличии магнитного поля $[16,17]$. Было установлено, что в графене доминирует электрон-электронное взаимодействие, которое является основным механизмом релаксации горячих носителей. В магнитных полях наблюдается увеличение времени релаксации, что объясняется подавлением релаксации из-за неэквидистантного расстояния между уровнями Ландау в графене. С ростом времени релаксации мы связываем увеличение ТГц фотопроводимости в графене (см. рис. 3). Магнитное поле оказывает значительное влияние на величину фотопроводимости начиная с некоторых критических его значений. Чем меньше концентрация электронов, тем более слабые магнитные поля обеспечивают рост сигнала фотопроводимости. При больших концентрациях электронов заполнены более высокие уровни Ландау, где энергия переходов между уровнями меньше из-за неэквидистатности уровней Ландау в графене. Поэтому в этом случае требуются более сильные магнитные поля для расщепления уровней Ландау по энергии и достижения некоторого предельного расстояния между уровнями, необходимого для подавления рассеяния. Расстояние между уровнями Ландау составляет несколько мэВ в условиях наших измерений (излучение с частотой $1-3$ ТГц и магнитные поля до 5Тл), что сравнимо с уширением уровней Ландау. Поэтому квантования уровней Ландау нет, так как они перекрываются, но при этом плотность состояний будет модулироваться. Понятно, что требуется достаточно сильная модуляция плотности состояний, чтобы влиять на эффективность электронных переходов в магнитном поле, что и определяет форму зависимости фотопроводимости от магнитного поля. Ранее при исследовании кинетики процессов релаксации в „рuтрprobe“ экспериментах наблюдалось увеличение времени релаксации, связанное с подавлением рекомбинации за счет электрон-электронного взаимодействия в графене в магнитном поле также в отсутствие квантования уровней Ландау [16].

Эффект магнитного поля на величину фотопроводимости может быть также связан с магнетосопротивлением. Как видно на рис. 2, $a$, с ростом магнитного поля сопротивление образца уменьшается. При этом может меняться и температурный коэффициент удельного сопротивления $\partial \rho_{x x} / \partial T_{L}$. По-видимому, этот эффект незначительный по сравнению с увеличением времени электрон-электронного рассеяния, поскольку не видно, как такой эффект может объяснить появление специфической формы зависимости $\Delta V$ от $B$, в частности не объясняет появление критических магнитных полей, $\mathrm{c}$ которых начитается рост фотопроводимости.

Кроме разогрева электронов при поглощении излучения, электроны могут нагреваться при пропускании тока через образец. Нагрев электронной системы при увеличении тока смещения приводит к появлению зависимости фотопроводимости $\Delta V$ от тока смещения $I_{d c}$. Как видно на рис. 3, $a$ и $c$, величина фотопроводимости в слабых магнитных полях значительно уменьшается при увеличении тока от 1.5 до 1.9 мкА. Об этом свидетельствует и сублинейная зависимость сигнала фотопроводимости от величины тока смещения (см. рис. 4), что можно объяснить разогревом двумерной электронной системы при протекании тока. Действительно, фотопроводимость

$$
\Delta V=I_{d c} \frac{L}{W} \frac{\partial \rho_{x x}}{\partial T_{L}} \Delta T_{e}
$$

определяется не только разогревом электронов $\Delta T_{e}$ терагерцовым излучением, но и джоулевым теплом, влияющим на температурный коэффициент $\frac{\partial \rho_{x x}}{\partial T_{L}}$. Отметим, что такой разогрев электронов из-за рассеиваемой мощности в образце приводит не только к значительному его нагреванию, но и, что важно, уменьшает вклад в разогрев электронов из-за поглощения излучения. Ясно, что раз излучение слабее влияет на нагрев образца, то и фотоответ в образце будет уменьшаться, что, соответственно, уменьшает чувствительность электронной системы к терагерцовому излучению. Этот эффект однозначно указывает на болометрический механизм фотопроводимости.

\section{6. Заключение}

В работе приведены результаты исследования терагерцовой фотопроводимости в эпитаксиальном графене, выращенном методом термической сублимации на поверхности 4H-SiC (1000). Исследования проводились на структурах в форме меандра, в котором отношение длины к ширине составляет 600. Проведены исследования фотоответа в магнитном поле при различных значениях концентрации электронов, тока смещения, интенсивности терагерцового излучения. Возникновение фотопроводимости объясняется разогревом носителей заряда в результате поглощения терагерцового излучения. Обнаружен сильный рост сигнала фотопроводимости вследствие ослабления электрон-электронного рассеяния в магнитном поле.

\section{Финансирование работы}

Работа выполнена при финансовой поддержке Foundation for Polish Science (IRA Program-CENTERA, grant MAB/2018/9, CENTERA), FLAG-ERA program (project 
DeMeGRaS, project GA501/16-1 of the DFG) и гранта Российского фонда фундаментальных исследований (18-02-00498).

\section{Конфликт интересов}

Авторы заявляют, что у них нет конфликта интересов.

\section{Список литературы}

[1] F.H.L. Koppens, T. Mueller, Ph. Avouris, A.C. Ferrari, M.S. Vitiello, M. Polini. Nature Nanotechnol., 9, 780 (2014).

[2] J. Yan, M.-H. Kim, J.A. Elle, A.B. Sushkov, G.S. Jenkins, H.M. Milchberg, M.S. Fuhrer, H.D. Drew. Nature Nanotechnol., 7, 472 (2012).

[3] Q. Han, T. Gao, R. Zhang, Y. Chen, J. Chen, G. Liu, Y. Zhang, Z. Liu, X. Wu, D. Yu. Sci. Rep., 3, 3533 (2013).

[4] Yu.B. Vasilyev, G.Yu. Vasileva, Yu.L. Ivanov, S. Novikov, S.N. Danilov. Appl. Phys. Lett., 105, 171105 (2014).

[5] G.Yu. Vasileva, D. Smirnov, Yu.B. Vasilyev, M.O. Nestoklon, N.S. Averkiev, S. Novikov, I.I. Kaya, R.J. Haug. Appl. Phys. Lett., 110, 113104 (2017).

[6] S.D. Ganichev, J. Diener, I.N. Yassievich, W. Prettl, B.K. Meyer, K.W. Benz. Phys. Rev. Lett., 75, 1590 (1995).

[7] P. Olbrich, J. Karch, E.L. Ivchenko, J. Kamann, B. März, M. Fehrenbacher, D. Weiss, S.D. Ganichev. Phys. Rev. B, 83, 165320 (2011).

[8] K.-M. Dantscher, D.A. Kozlov, P. Olbrich, C. Zoth, P. Faltermeier, M. Lindner, G.V. Budkin, S.A. Tarasenko, V.V. Belkov, Z.D. Kvon, N.N. Mikhailov, S.A. Dvoretsky, D. Weiss, B. Jenichen, S.D. Ganichev. Phys. Rev. B, 92, 165314 (2015).

[9] S.D. Ganichev, Ya.V. Terent'ev, I.D. Yaroshetskii. Sov. Tech. Phys. Lett., 11, 20 (1985).

[10] C. Drexler, N. Dyakonova, P. Olbrich, J. Karch, M. Schafberger, K. Karpierz, Yu. Mityagin, M.B. Lifshits, F. Teppe, O. Klimenko, Y.M. Meziani, W. Knap, S.D. Ganichev. J. Appl. Phys., 111, 124504 (2012).

[11] S. Kopylov, A. Tzalenchuk, S. Kubatkin, V.I. Fal'ko. Appl. Phys. Lett., 97, 112109 (2010).

[12] S. Lara-Avila, K. Moth-Poulsen, R. Yakimova, T. Bjørnholm, V. Fal'ko, A. Tzalenchuk, S. Kubatkin. Adv. Mater., 23, 878 (2011).

[13] Y. Kawano, Y. Hisanaga, H. Takenouchi, S. Komiyama. J. Appl. Phys., 89, 4037 (2001).

[14] D. Sun, Z.K. Wu, C. Divin, X. Li, C. Berger, W.A. deHeer, P.N. First, T.B. Norris. Phys. Rev. Lett., 101, 157402 (2008).

[15] K.J. Tielrooij, J.C.W. Song, S.A. Jensen, A. Centeno, A. Pesquera, A. Zurutuza Elorza, M. Bonn, L.S. Levitov, F.H.L. Koppens. Nature Phys., 9, 248 (2013).

[16] P. Plochocka, P. Kossacki, A. Golnik, T. Kazimierczuk, C. Berger, W.A. de Heer, M. Potemski. Phys. Rev. B, 80, 245415 (2009).

[17] M. Mittendorff, F. Wendler, E. Malic, A. Knorr, M. Orlita, M. Potemski, C. Berger, W.A. de Heer, H. Schneider, M. Helm. Nature Phys., 11, 75 (2015).

Редактор Г.А. Оганесян

\section{Terahertz photoconductivity in graphene in a magnetic field}

\author{
Yu.B. Vasiliev' ${ }^{1}$, S.N. Novikov², S.N. Danilov ${ }^{3}$, \\ S.D. Ganichev ${ }^{3,4}$ \\ ${ }^{1}$ loffe Institute, \\ 194021 St. Petersburg, Russia \\ 2 Aalto University, \\ 02150 Espoo, Finland \\ ${ }^{3}$ Terahertz Center TerZ, University of Regensburg, \\ Regensburg, Germany \\ ${ }^{4}$ CENTERA Laboratories, Institute of High Pressure \\ Physics, Polish Academy of Sciences, \\ 01-142 Warsaw, Poland
}

\begin{abstract}
We study terahertz photoconductivity in a magnetic field in epitaxial graphene grown on $\mathrm{SiC}$ substrates. The dependence of the amplitude of the photoresponse signal on the magnitude of the magnetic field was studied for different values of electron concentration, bias current, and terahertz radiation intensity. The photoconductivity mechanism based on the heating of electrons by terahertz radiation well explains the experimental results. With increasing magnetic field, a strong increase in the photoconductivity signal was detected due to an increase in the relaxation time due to suppression of electron-electron scattering.
\end{abstract}

